\title{
Nanocompósitos de PVC com Argila Organicamente Modificada: Efeitos do Processamento e do Método de Incorporação da Argila
}

Antonio Rodolfo Jr. Braskem S/A

Lucia H. I. Mei Departamento de Tecnologia de Polímeros, Faculdade de Engenharia Química, Universidade Estadual de Campinas

Resumo: Nanocompósitos de poli(cloreto de vinila) (PVC) flexível com argila organicamente modificada (O-MMT) foram preparados utilizando-se diferentes metodologias de produção, de forma a estudar seus efeitos sobre o grau de intercalação/ esfoliação da argila. A morfologia dos nanocompósitos obtidos foi avaliada através de difratometria de raios X (DRX) e microscopia eletrônica de transmissão (MET). Foram também avaliadas propriedades de tração e estabilidade térmica estática das formulações preparadas. Nanocompósitos de microestrutura híbrida intercalada/parcialmente esfoliada foram obtidos, independentemente do composto de PVC ter sido processado a partir do pó ou granulado (extrudado previamente), e do método de incorporação da O-MMT à resina de PVC (diretamente no misturador intensivo ou pré-esfoliada em DIDP/OSE a quente). Os plastificantes, presentes na formulação do composto de PVC flexível, exerceram um papel importante no processo de intercalação e esfoliação da argila, facilitando a formação dos nanocompósitos.

Palavras-chave: $P V C$, poli(cloreto de vinila), nanocompósito polímero-argila.

\section{PVC/Organically Modified Montmorillonite Nanocomposites: Effects of Processing and Clay Incorporation Methodology}

Abstract: Nanocomposites of poly(vinyl chloride) (PVC) and an organically modified montmorillonite (O-MMT) were prepared using different production approaches to assess possible effects on the degree of clay intercalation/exfoliation. The morphology of the nanocomposites was studied using X ray diffratometry and transmission electron microscopy. Tensile properties and static thermal stability were also evaluated. Hybrid intercalated/partially exfoliated nanocomposites were obtained, regardless of whether the PVC compound was processed from the powder (dry blend) or granulated (extruded) samples, or of the methodology used for incorporation of O-MMT into the PVC compound (directly in the intensive mixer or pre-exfoliated in a hot mixture of DIDP/ESO). The plasticizers, present in the flexible PVC compound, exerted an important role in the process of intercalation and exfoliation of the clay, helping the formation of the nanocomposites.

Keywords: PVC, poly(vinyl chloride), polymer/clay nanocomposites.

\section{Introdução}

O PVC, ou poli(cloreto de vinila), é o segundo termoplástico mais consumido em todo o mundo, com uma demanda mundial de resina superior a 36 milhões de toneladas no ano de 2008, sendo a capacidade mundial de produção de resinas de PVC estimada em cerca de 43 milhões t/ano ${ }^{[1]}$. O Brasil foi responsável pelo consumo de cerca de $970 \mathrm{mil}$ toneladas, ou 2,7\% da demanda mundial de resinas de PVC. Estes dados mostram o potencial de crescimento da demanda destas resinas no Brasil, uma vez que o consumo per capita, na faixa de $5 \mathrm{~kg} / \mathrm{hab} / \mathrm{ano}$, ainda é baixo comparado com estes e outros países.
O PVC pode ser considerado um dos mais versáteis dentre os plásticos. Devido à necessidade da resina ser formulada mediante a incorporação de aditivos, o PVC pode ter suas características alteradas dentro de um amplo espectro de propriedades, em função da aplicação final, variando desde o rígido ao extremamente flexível. Esta grande faixa de variação de propriedades permite que o PVC seja utilizado em aplicações que vão desde tubos e perfis rígidos, para uso na construção civil, até brinquedos e laminados flexíveis para acondicionamento de sangue e plasma. A grande versatilidade do PVC deve-se também, em parte, à sua adequação aos mais variados processos de moldagem, podendo o mesmo ser injetado, extrudado, calandrado, espalmado,

Autor para correspondência: Antonio Rodolfo Jr., Braskem S/A, Edifício Eldorado Business Tower, Av. das Nações Unidas 8501, 23ำ andar, 
somente para citar algumas das alternativas de transformação.

Nanocompósitos poliméricos são uma nova classe de compósitos que contém pequenas quantidades, em geral abaixo de 5\% em massa, de nanopartículas de reforço, com ao menos uma de suas dimensões da ordem de grandeza do nanômetro. Ou seja, tipicamente elas medem entre $1 \mathrm{e} 100 \mathrm{~nm}^{[2,3]}$. Quanto à natureza, as partículas podem ser de escala zero-dimensional (nanopartícula), unidimensional (nanofibra) e bidimensional (nanoplaquetas), em função da quantidade de dimensões em escala nanométrica contidas na mesma $^{[4]}$.

Nas últimas décadas, com o desenvolvimento da nanotecnologia, tem havido um interesse crescente no campo dos nanocompósitos poliméricos, devido à suas propriedades especiais. Além de possibilitarem a obtenção das propriedades equivalentes à dos compósitos tradicionais, exibem adicionalmente propriedades ópticas, elétricas e magnéticas muitas vezes únicas ${ }^{[5]}$.

De acordo com Gilman ${ }^{[6]}$, nanocompósitos de polímeros com argilas foram inicialmente reportados em 1961, quando Blumstein demonstrou que monômeros vinílicos polimerizavam-se de forma intercalada nas galerias de argilas do tipo montmorilonita. A primeira aplicação comercial, segundo Giannelis ${ }^{[7]}$, foi viabilizada pela Toyota no Japão, no início dos anos 1990, e consistia de um nanocompósito de poliamida 6 com montmorilonita. Ainda segundo este autor, dois pesquisadores japoneses, Fujiwara e Sakamoto, foram os primeiros a reportarem as propriedades potenciais de retardância à chama desta nova classe de materiais, em uma patente 1976.

Os nanocompósitos poliméricos apresentam propriedades mecânicas e térmicas superiores aos compósitos convencionais, mesmo com uma quantidade menor de reforço, devido à área de contato maior entre o polímero e a fase neste dispersa. Ademais, o elevado fator de forma dos reforços incorporados, também denominado razão de aspecto, propicia importantes propriedades de barreira na maioria dos casos. A baixa permeabilidade, melhor resistência química e maior retardância de chama são atribuídas às melhores propriedades de barreira dos nanocompósitos. O caminho para obtenção de tal desempenho consiste na habilidade de dispersar, individualmente, estas partículas com elevado fator de forma em meio à matriz polimérica ${ }^{[8,9]}$.

Os reforços mais comumente utilizados para a produção de nanocompósitos poliméricos são as argilas, que sendo da família dos minerais, possuem uma constituição química que lhes permite a separação das camadas de silicato (esfoliação), com consequente possibilidade de intercalação das cadeias poliméricas. A propriedade de possuir elevada área superficial faz com que as argilas tragam uma série de benefícios potenciais aos materiais poliméricos, incluindo o PVC; ou sejam, maior rigidez e resistência mecânica, maior tenacidade, maior barreira à difusão de gases, menor permeabilidade, maiores temperaturas de distorção e amolecimento, menor inflamabilidade, melhor resistência química e maior estabilidade dimensional ${ }^{[10-14]}$.

Uma troca de cátions interlamelares, por sais de amônio quaternário, origina a argila organofílica, reduzindo a energia de superfície das camadas da argila. Isto então torna possível que espécies orgânicas se difundam ou penetrem entre as camadas, eventualmente separando-as ou esfoliando-as. Embora estes agentes de modificação tenham conquistado sucesso significativo na preparação de nanocompósitos polímero-argila, seu principal problema é a estabilidade térmica. Como muitos compósitos poliméricos são preparados e intercalados no estado fundido, em temperaturas elevadas, a estabilidade térmica do modificador orgânico é de extrema importância. Todos os componentes dos nanocompósitos podem estar sujeitos a altas temperaturas durante o processo de preparação. Entretanto, se a temperatura de processamento for maior que a estabilidade térmica da argila organofílica, a decomposição desta ocorrerá, e a interface entre o reforço e a matriz polimérica será efetivamente alterada.

No caso particular do PVC, entretanto, o maior problema decorrente do uso de sais de amônio quaternário é seu forte caráter de ácido de Lewis. Este efeito é fortemente deletério para este polímero, promovendo sua rápida degradação térmica pelo mecanismo de desidrocloração. Tal fato, indesejado, não pode ser ignorado na produção de nanocompósitos de PVC com argilas organicamente modificadas ${ }^{[15]}$.

A maior concentração de artigos publicados a respeito de nanocompósitos à base de PVC tem por objetivo a intercalação de argilas, fundamentalmente montmorilonita (MMT). Os artigos, que relatam processos de intercalação ou mistura no estado fundido, são também mais freqüentes. Isto é compreensível, sob o ponto de vista experimental, uma vez que a polimerização do cloreto de vinila, que é um gás à temperatura e pressão ambientes, é complexa do ponto de vista prático. Tal fato tem sido o responsável pelo número reduzido de laboratórios acadêmicos que trabalham neste campo, particularmente em virtude do fator toxicidade do monômero. Por sua vez, o processo de intercalação ou mistura no estado fundido requer equipamentos mais simples e acessíveis como extrusoras, reômetros de torque ou moinhos de rolos. Este processo não necessita tanta preocupação com o processo de polimerização, o qual nem sempre está disponível a todos os grupos de pesquisa.

Wang e colaboradores ${ }^{[16]}$ realizaram estudos de intercalação de uma montmorilonita organicamente modificada (O-MMT) (Cloisite ${ }^{\circledR}$ 30B, Southern Clay Products Inc.) em PVC, com e sem a presença de dioctilftalato (DOP) como plastificante. A intercalação no estado fundido foi realizada em um reômetro de torque Brabender, o que possibilitou a obtenção de nanocompósitos de PVC/O-MMT intercalados e parcialmente esfoliados, conforme indicativos de difratometria de raios $\mathrm{X}(\mathrm{DRX})$. As propriedades mecânicas e térmicas foram muito discretamente alteradas, indicando que a argila pode ter agido na verdade como carga e não como reforço da matriz polimérica. Uma suspeita dos autores é de que os 
resultados, apresentados somente como médias das propriedades, sem os respectivos desvios, podem ser estatisticamente insignificantes, uma vez que as fotomicrografias de microscopia eletrônica de transmissão (MET), apresentadas no trabalho, mostram aglomerados de argila, reconhecidamente fragilizadores em compósitos poliméricos ${ }^{[17]}$.

Yalcin e Cakmak ${ }^{[18]}$ avaliaram os efeitos do DOP como auxiliar no processo de esfoliação de uma O-MMT (Cloisite ${ }^{\circledR}$ 30B), em meio à matriz de PVC. Os nanocompósitos foram obtidos em duas etapas; na primeira, a O-MMT foi misturada ao DOP e ao estabilizante térmico líquido à base de estanho, à temperatura ambiente; na segunda, esta mistura foi incorporada a um composto de PVC comercial em um reômetro de torque Brabender. Os resultados obtidos por estes dois pesquisadores são interessantes e mostram que foram conseguidos nanocompósitos de estrutura híbrida intercalada/parcialmente esfoliada, demonstrado tanto por DRX quanto por MET. $\mathrm{O}$ aumento do teor de plastificante é um dos fatores chave deste processo, uma vez que foi observado que maiores teores de DOP facilitam o processo de intercalação. Porém, eles reduzem a qualidade da dispersão dos aglomerados pela menor eficiência de transferência de tensões sob cisalhamento, devido à menor viscosidade do sistema, tal como observado por microscopia de força atômica (AFM). Desta forma, um balanço entre as duas características deve ser conseguido, de forma a otimizar as propriedades finais do nanocompósito. Os autores não apresentam resultados de propriedades mecânicas ou térmicas de seus nanocompósitos, nem discutem sobre os efeitos da O-MMT na estabilidade térmica ou coloração do produto final.

A abordagem de se utilizar o DOP como agente intercalador da argila foi também explorada por Kovarova e colaboradores ${ }^{[19]}$. Utilizando-se de dois tipos de argila (montmorilonita sódica ou Na-MMT e O-MMT Cloisite ${ }^{\circledR}$ 30B), prepararam uma mistura das mesmas com DOP por 30 minutos a $80^{\circ} \mathrm{C}$. Em seguida, esta mistura foi incorporada à resina de PVC e demais aditivos da formulação e processadas em uma extrusora com forte poder de mistura (Buss Co-Kneader). Os nanocompósitos foram passados até duas vezes pela extrusora de forma a permitir a avaliação dos efeitos do processamento na qualidade dos mesmos. Os pesquisadores limitaramse a avaliar a dispersão, intercalação e possível esfoliação das argilas na matriz de PVC, e destacam os benefícios de seu processo de pré-tratamento da argila. Porém, o trabalho não oferece subsídios comparativos de formulações sem o uso desta etapa prévia ao processamento, para averiguação dos seus reais efeitos. De qualquer forma, foram obtidos nanocompósitos desordenados e parcialmente intercalados no caso da Na-MMT, e intercalados/parcialmente esfoliados no caso da O-MMT, nas condições de processamento consideradas. O estudo também destaca que a solução proposta protege a matriz de PVC dos efeitos deletérios do sal de amônio quaternário, pela barreira formada pelo DOP decorrente do pré-tratamento; porém, novamente não foram fornecidos subsídios para comprovar tal fato.
Peprnicek e colaboradores ${ }^{[2,21]}$ publicaram recentemente dois estudos sobre nanocompósitos PVC/O-MMT. No primeiro estudo, foram avaliados os efeitos do cisalhamento e da temperatura em nanocompósitos de PVC obtidos pela intercalação no estado fundido. Foram testadas três argilas comercialmente disponíveis (Na-MMT e O-MMT Cloisite $^{\circledast}$ 30B e Cloisite ${ }^{\circledR}$ 93A), com diferentes tipos de sal de amônio quaternário. A preparação dos nanocompósitos se deu pela dispersão da argila em DOP, na concentração de $5 \%$, usando um misturador intensivo por 15 minutos, à temperatura ambiente. Simultaneamente, foi também incorporado o efeito da temperatura a esta etapa de pré-esfoliação da argila sob cisalhamento no misturador intensivo, através do aquecimento da mistura DOP/argila a $130{ }^{\circ} \mathrm{C}$. Neste estudo o grupo utilizou uma resina de PVC do tipo micro-suspensão, a qual formou uma pasta de PVC (plastissol) com a adição da mistura de DOP/argila e demais aditivos, que foi gelificada/ fundida a $170{ }^{\circ} \mathrm{C}$, por 8 minutos, sem cisalhamento adicional (em repouso). Resultados de DRX, segundo os autores, dão indicativos de que o processo envolvendo temperatura e cisalhamento é mais eficiente na dispersão e intercalação/esfoliação das argilas na matriz de PVC; porém, um exame cuidadoso dos difratogramas apresentados na publicação permite somente esta conclusão para o caso da Na-MMT. Nos casos das O-MMT, fica difícil chegarmos a esta conclusão, uma vez que o artigo não traz micrografias de MET para suportar as conclusões dos autores. Este estudo permitiu evidenciar um efeito bastante pronunciado na estabilidade térmica dos nanocompósitos, medida em estufa com circulação de ar. A presença e o tipo de sal de amônio quaternário influenciam fortemente na estabilidade térmica da matriz de PVC, com um efeito bastante negativo no caso da argila Cloisite ${ }^{\circledR}$ 93A. Infelizmente, maiores níveis de esfoliação (segundo os autores) foram obtidos na argila com pior efeito sobre a estabilidade térmica do PVC, o que reforça a necessidade de aprofundamento no desenvolvimento de argilas organofílicas específicas para o PVC.

Francis e Schmidt ${ }^{[22]}$ estudaram a formação de nanocompósitos flexíveis de PVC (na forma de composto pronto, grau médico, plastificado com DOP) e O-MMT (Cloisite ${ }^{\circledR}$ 30B), em uma extrusora de roscas duplas Werner \& Pfleiderer $30 \mathrm{~mm}$. A argila em pó foi misturada diretamente aos grânulos do composto de PVC em um misturador intensivo, por 15 minutos, previamente à extrusão e injeção de corpos de prova. Mesmo nestas condições, aparentemente desfavoráveis, foram obtidos nanocompósitos de PVC de estrutura híbrida intercalada/parcialmente esfoliada.

Outro grupo de pesquisadores, liderado por Kalendova ${ }^{[23]}$, avaliou a incorporação de Na-MMTe O-MMT (Cloisite ${ }^{\circledR}$ 30B) em um composto de PVC flexível. As argilas foram pré-esfoliadas em plastificante a $80{ }^{\circ} \mathrm{C}$, seguido de incorporação ao composto de PVC e intercalação no estado fundido, em um misturador Buss de alto cisalhamento. Neste estudo foi possível observar melhoria das propriedades de combustão das amostras, obtidas por calandragem em um moinho de ro- 
los, contendo 5\% de MMT vs. o composto de PVC tomado como referência. Os autores atribuíram os resultados obtidos à formação de um resíduo carbonáceo-silicoso na superfície das amostras durante a combustão.

\section{Experimental}

\section{Materiais}

A formulação do composto de PVC, tomada como referência e resumida na Tabela 1 , baseou-se nas práticas comuns utilizadas nos transformadores de fios e cabos de PVC. Resina de PVC com valor K $65 \pm 1$ (Norvic ${ }^{\circledR}$ SP 1000 , fornecida pela Braskem S/A) foi utilizada como matriz polimérica principal. A estabilização térmica da formulação, sob ação da temperatura e cisalhamento presentes no processamento, foi efetuada através da incorporação de $\mathrm{Ca} / \mathrm{Zn}$ (Naftomix ${ }^{\circledR}$ XC-1202, fornecido pela Chemson Ltda.).

A flexibilização da formulação foi conseguida pelo uso tanto de diisodecilftalato (DIDP) (Scandiflex do Brasil S/A Indústrias Químicas), quanto óleo de soja epoxidado (OSE) (Drapex ${ }^{\circledR}$ 6.8, Inbra Indústrias Químicas Ltda.), respectivamente, plastificantes primário e secundário. O OSE possui efeito sinergístico com os carboxilatos de $\mathrm{Ca}$ e $\mathrm{Zn}$ do estabilizante térmico, atuando portanto como um coestabilizante ${ }^{[15]}$.

Para manter coerência com as formulações tipicamente utilizadas pela indústria de compostos de PVC para isolamento de fios e cabos elétricos, foi incorporado carbonato de cálcio precipitado (Barralev ${ }^{\circledR} \mathrm{C}$, Imerys do Brasil Mineração Ltda.). Um lubrificante externo (ácido esteárico, Chemson Ltda.), necessário ao adequado processamento da formulação nos diversos equipamentos utilizados, também foi incorporado.

\section{Argila}

A argila utilizada neste estudo foi a Cloisite ${ }^{\circledR} 30 \mathrm{~B}$, fornecida pela Southern Clay Minerals, Inc. (EUA), e amplamente utilizada em diversos outros estudos envolvendo a obtenção de nanocompósitos de PVC ${ }^{[16,18-21]}$. Trata-se de uma montmorilonita natural, modificada com um sal de amônio quaternário baseado em metil-2-hidroxietil, acoplado a uma cadeia graxa de origem animal (sebo), contendo aproximadamente $65 \% \mathrm{C}_{18}$, $30 \% \mathrm{C}_{16}$ e $5 \% \mathrm{C}_{14}{ }^{[24]}$. Esta argila possui, como referência, um valor de espaçamento interlamelar $\left(\mathrm{d}_{001}\right)$ igual a $1,85 \mathrm{~nm}$ (DRX), e foi incorporada na dosagem de $5 \%$ em massa.

Tabela 1. Formulação do composto de PVC utilizado neste estudo.

\begin{tabular}{cc}
\hline Componente & Teor (pcr) \\
\hline Norvic $^{\circledR}$ SP 1000 & 100 \\
Naftomix $^{\circledR}$ XC-1202 & 3,5 \\
DIDP & 45 \\
Drapex $^{\circledR} 6.8$ & 5 \\
Barralev $^{\circledR}$ C & 40 \\
Ácido esteárico & 0,2 \\
\hline
\end{tabular}

\section{Preparação dos compostos}

Diferentes abordagens experimentais foram adotadas na preparação de seis compostos de PVC, de forma a permitir a avaliação de seus efeitos na morfologia dos nanocompósitos resultantes.

Todas as formulações foram homogeneizadas, utilizandose um misturador intensivo Mecanoplast ML-9. Inicialmente a resina, o estabilizante térmico, o carbonato de cálcio e o ácido esteárico foram adicionados ao misturador, à temperatura ambiente, e após cisalhamento sob temperatura de $80^{\circ} \mathrm{C}$, foram incorporados os plastificantes DIDP e OSE, bem como a argila nos casos aplicáveis. A descarga da composição final foi realizada a $110{ }^{\circ} \mathrm{C}$, seguida de resfriamento até $35-40{ }^{\circ} \mathrm{C}$ para evitar a formação de aglomerados.

Um dos efeitos avaliados neste estudo foi a pré-intercalação/esfoliação da argila em DIDP/OSE. Para isto, a quantidade de plastificante necessária à cada formulação do composto, foi aquecida a $90-100{ }^{\circ} \mathrm{C}$ em um béquer, utilizando-se de uma placa de aquecimento. A argila necessária para compor a formulação foi cisalhada à quente, na presença dos plastificantes, utilizando-se de um agitador Fisatom 7137 (70 W), a 1200-1300 rpm, por 20 minutos. Esta alternativa de processo de produção dos nanocompósitos foi comparada com a opção de incorporação da argila diretamente no misturador intensivo, na temperatura de $100{ }^{\circ} \mathrm{C}$ e pouco antes da descarga da mistura.

Outro efeito avaliado neste estudo foi a influência da granulação do composto obtido na forma de pó, ou dry blend, ao final do ciclo de mistura descrito anteriormente. Algumas das formulações foram processadas em uma extrusora Miotto modelo LM 03/30 (30 mm, L/D 25), usando-se o perfil de temperatura de $140-145-150{ }^{\circ} \mathrm{C}$ e $80 \mathrm{rpm}$. A Tabela 2 apresenta de maneira resumida os diferentes fatores estudados.

Em todos os casos, independentemente do processo de produção escolhido, corpos-de-prova foram obtidos através da plastificação das composições, em uma calandra de laboratório Mecanoplast. A temperatura, o tempo e a rotação de operação para preparar as placas de cerca de $3 \mathrm{~mm}$ de espessura com as quais se confeccionou os corpos-de-prova foram $160{ }^{\circ} \mathrm{C}$, por 3 minutos a $20 \mathrm{rpm}$, respectivamente. A prensagem das chapas foi feita entre placas de aço inoxidável, em uma prensa Luxor a $175^{\circ} \mathrm{C}$.

Tabela 2. Sumário dos fatores estudados nas diferentes formulações de PVC/O-MMT preparadas.

\begin{tabular}{cccc}
\hline Formulação & $\begin{array}{c}\text { Teor de } \\
\text { O-MMT }(\%)\end{array}$ & $\begin{array}{c}\text { Pré-esfoliação } \\
\text { a quente }\end{array}$ & Granulação \\
\hline F1 & 0 & - & Não \\
F2 & 0 & - & Sim \\
F3 & 5 & Não & Não \\
F4 & 5 & Não & Sim \\
F5 & 5 & Sim & Não \\
F6 & 5 & Sim & Sim \\
\hline
\end{tabular}




\section{Avaliação morfológica dos nanocompósitos obtidos}

A formação dos nanocompósitos foi avaliada através de técnicas consideradas complementares ${ }^{[12,13]}$, como DRX e MET. Difratogramas de raios $X$ foram obtidos em amostras retiradas diretamente do filme calandrado, em um difratômetro Siemens D500, no modo de reflexão, utilizando-se de um feixe de raios $\mathrm{X}$ incidente de $\mathrm{Cu} \mathrm{K} \alpha(\lambda=1,54 \AA)$. Foram realizadas varreduras entre $2 \theta=1 \mathrm{e} 45^{\circ}$, com passo de $0,05^{\circ}$.

Amostras finas (cerca de $70 \mathrm{~nm}$ de espessura) de cada formulação foram obtidas em um ultramicrótomo Leica EM FC6, com faca de diamante Drukker $3 \mathrm{~mm} / 45^{\circ}$, na temperatura de $-140{ }^{\circ} \mathrm{C}$. As observações de MET destas amostras finas foram realizadas em um equipamento Jeol JEM-1200 EX II, a $80 \mathrm{kV}$.

\section{Propriedades mecânicas}

Propriedades de tração (módulo de Young, tensão de ruptura e alongamento na ruptura) foram determinadas em oito corpos de prova ASTM D638-08 tipo IV, para cada formulação, em uma máquina universal de ensaios MTS modelo Alliance 5/RT, na velocidade de $50 \mathrm{~mm} / \mathrm{min}$. Os ensaios foram realizados na temperatura de $(23 \pm 2){ }^{\circ} \mathrm{C}$.

\section{Estabilidade térmica}

A estabilidade térmica estática das diferentes formulações foi determinada em um forno Metrastat IR 400, com exposição de 1 hora nas temperaturas de 190,210 e $230{ }^{\circ} \mathrm{C}$. As fitas resultantes da exposição à temperatura no forno Metrastat foram analisadas quanto à variação de cor $\left(\Delta \mathrm{E} \mathrm{L} \mathrm{a}^{*} \mathrm{~b} *\right)$ através de scanner e software FluoScan (Dr. Stapfler GmbH, Alemanha).

\section{Resultados e Discussão}

\section{Avaliação morfológica dos nanocompósitos obtidos}

As Figuras 1 e 2 apresentam os resultados de DRX das formulações contendo O-MMT em sua composição. É possível verificar que, independentemente do processo de pro-

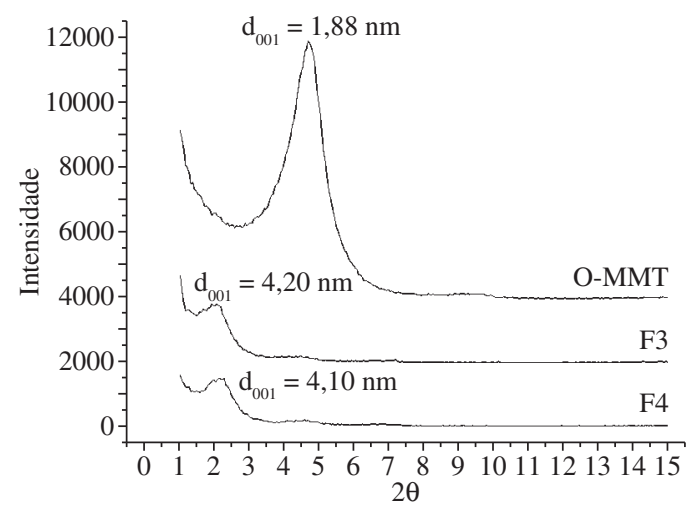

Figura 1. Difratogramas de raio $X$ para as amostras de nanocompósitos PVC/O-MMT obtidos pela incorporação da argila diretamente no misturador intensivo: F3 (composto em pó); e F4 (composto granulado).

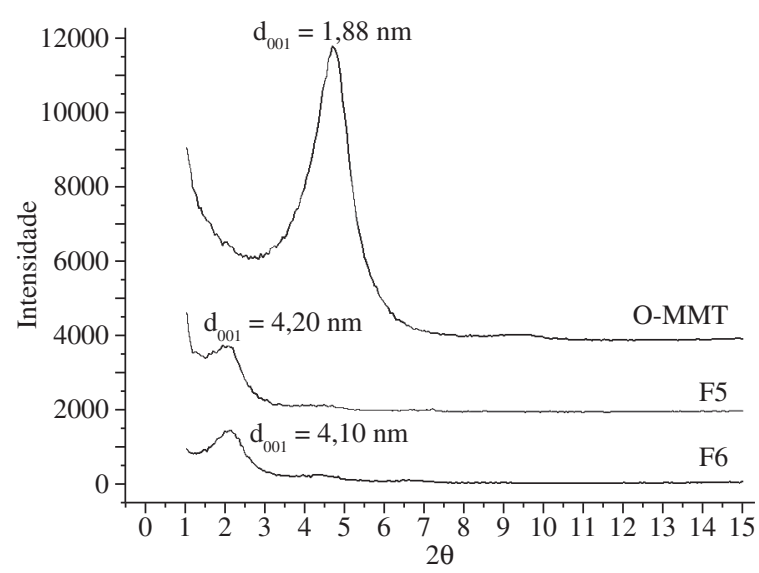

Figura 2. Difratogramas de raio $X$ para as amostras de nanocompósitos PVC/O-MMT obtidos pela incorporação da argila previamente esfoliada em DIDP/OSE a quente: F5 (composto em pó); e F6 (composto granulado).

dução escolhido, ocorreu a formação de nanocompósitos de PVC/O-MMT, com estrutura híbrida intercalada/parcialmente esfoliada. Em todos os casos ocorreu deslocamento e atenuação do pico $\mathrm{d}_{001}$ da O-MMT, originalmente a $2 \theta=4,70^{\circ}$ $\left(\mathrm{d}_{001}=1,88 \mathrm{~nm}\right.$, em linha com os dados do fornecedor $\left.{ }^{[24]}\right)$, para valores da ordem de $2 \theta=2,10$ a $2,15^{\circ}\left(d_{001}=4,10\right.$ a $\left.4,20 \mathrm{~nm}\right)$. Este deslocamento do pico de difração principal da O-MMT, para valores menores de ângulo $2 \theta$, é um importante indicativo do aumento do espaçamento entre as lamelas do silicato, resultante da intercalação de moléculas dos plastificantes e/ou de cadeias do PVC nas galerias da argila. Resultados de outros autores ${ }^{[16,18-23]}$, sempre utilizando DOP como plastificante, indicam valores diversos para $\mathrm{d}_{001}$, variando entre 3,1 até $4,9 \mathrm{~nm}$; ou seja, da mesma ordem de grandeza que os obtidos neste estudo.

O processo de gelificação e fusão das resinas de PVC durante o processamento é um fenômeno bastante complexo, do ponto de vista de sua cinética, e foi amplamente estudado $^{[25-29]}$. De maneira resumida, trata-se da destruição dos grãos de PVC (120-150 $\mu \mathrm{m}$ de diâmetro) originários do processo de polimerização em suspensão, por conta de cisalhamento. Como consequência, ocorrerá a formação de um gel de partículas primárias ( $\sim \mu \mathrm{m}$ de diâmetro), fundido pela interdifusão de macromoléculas decorrente dos efeitos do cisalhamento e da temperatura. Neste contexto, acreditamos ser pouco eficiente a intercalação de macromoléculas de PVC nas galerias do silicato, uma vez que seria termodinâmicamente mais favorável a intercalação de moléculas orgânicas de baixo peso molecular, tal como as do plastificante, que estariam promovendo o observado aumento do espaçamento interlamelar identificado nas medidas de DRX.

Os difratogramas apresentados sugerem ainda que, mesmo após o processamento, existem ainda evidências da presença de tactóides (aglomerados não esfoliados) remanescentes de intercalação/esfoliação da O-MMT, demonstrado pela presença de picos bastante discretos e largos ao redor de $2 \theta=4,70^{\circ}$. 


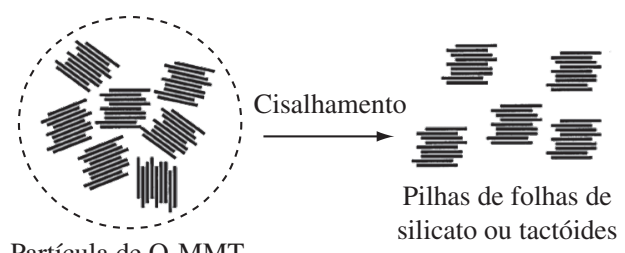

Partícula de O-MMT $(\sim 8 \mu \mathrm{m})$

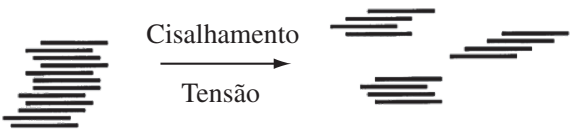

Cisalhamento das pilhas de plaquetas leva a menores tactóides

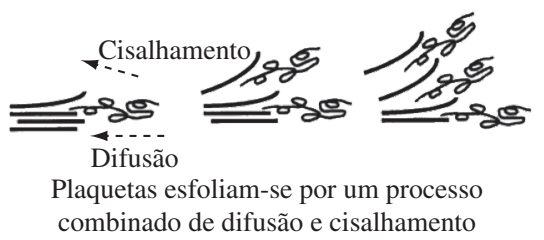

Figura 3. Mecanismo de dispersão e esfoliação da argila durante o processo de intercalação no estado fundido, segundo Fornes e colaboradores ${ }^{[25]}$.

Paul e Robeson ${ }^{[12]}$ citam que este tipo de coexistência de diferentes níveis de esfoliação, desde totalmente não esfoliado (tactóides) até a esfoliação total (lamelas de silicato dispersas na matriz polimérica), é comum em nanocompósitos obtidos pelo processo de intercalação no estado fundido. Isto ocorre em função do próprio mecanismo de dispersão e esfoliação da argila, apresentado de maneira esquemática na Figura 3. O mecanismo proposto por Fornes e colaboradores ${ }^{[30]}$ sugere que o processo de esfoliação da argila inicia-se com a divisão das partículas originais do O-MMT em tactóides. Tais aglomerados são posteriormente deformados por conta da taxa e tensão de cisalhamento incidentes, durante o processamento no estado fundido. Finalmente, por conta de um processo combinado de difusão de cadeias e de outros componentes orgânicos da formulação, como os plastificantes, e de cisalhamento resultante do processamento, as folhas ou lamelas de silicato sofrem a intercalação nas galerias, podendo até atingir uma esfoliação completa.

As Figuras 4 e 5 complementam as constatações da análise de DRX. As observações de MET permitem visualizar em todas as amostras a presença de folhas de silicato esfoliadas, coexistindo com estruturas intercaladas e mesmo alguns tactóides, confirmando as evidências demonstradas na análise de DRX.

\section{Propriedades mecânicas}

As Figuras 6, 7 e 8 apresentam, respectivamente, os resultados de módulo de Young, tensão de ruptura e alongamento na ruptura, das diferentes formulações estudadas. É possível verificar que, em todas estas propriedades, houve um efeito significativo com a incorporação da O-MMT, em comparação com as formulações F1 e F2 tomadas como referência. A incorporação da argila promove aumento do módulo de

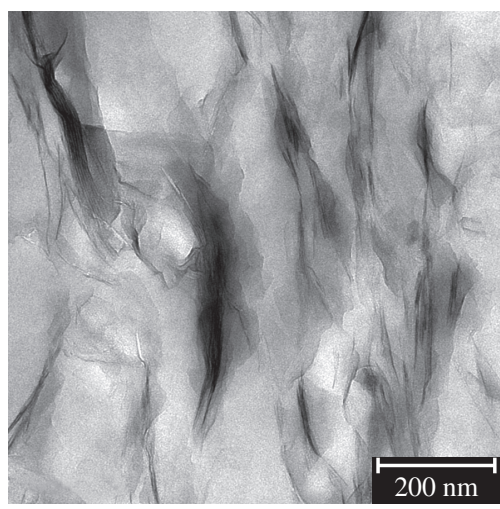

(a)

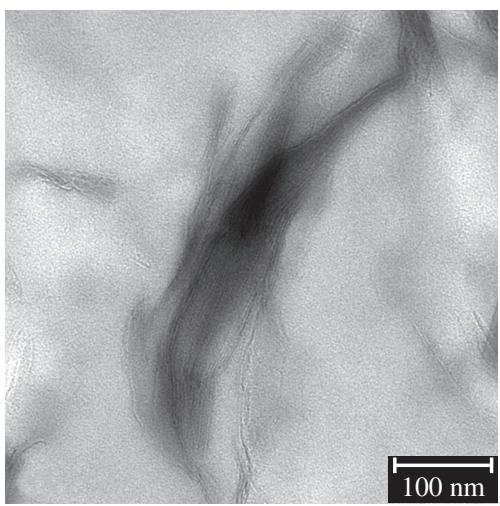

(b)

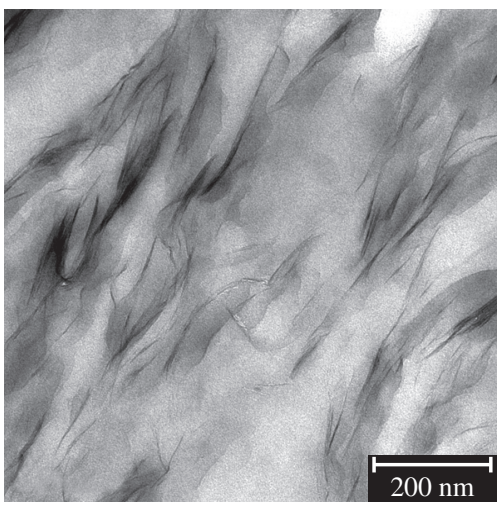

(c)

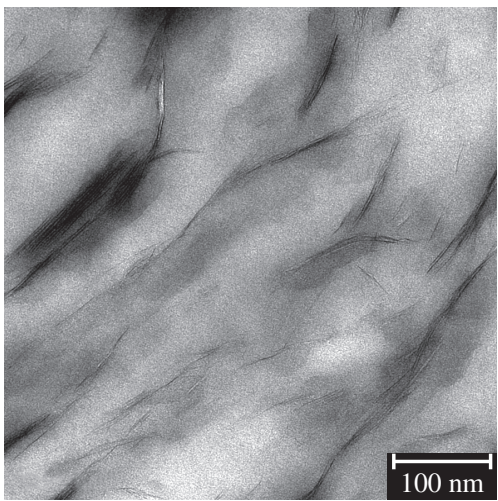

(d)

Figura 4. Fotomicrografias de MET para as amostras de nanocompósitos PVC/O-MMT obtidos pela incorporação da argila diretamente no misturador intensivo: a) e b) F3 (composto em pó); c) e d) F4 (composto granulado). 


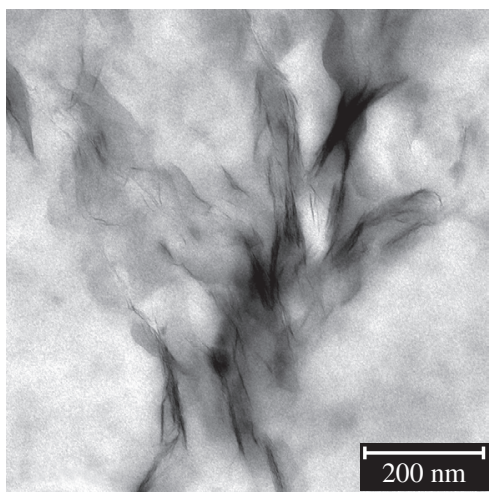

(a)

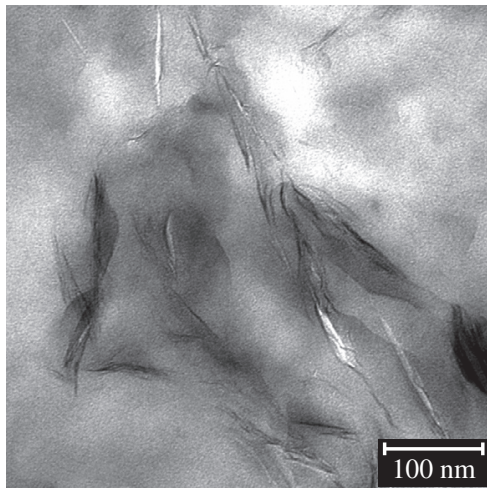

(b)

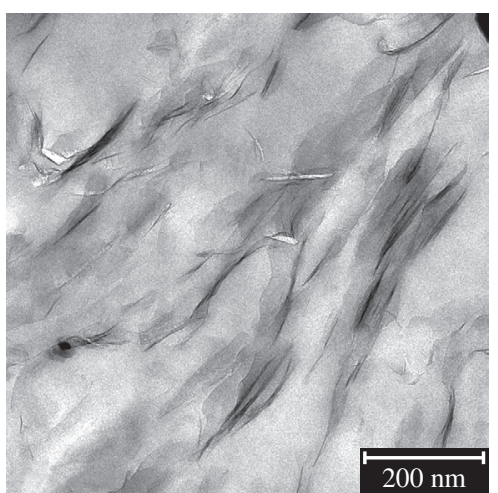

(c)

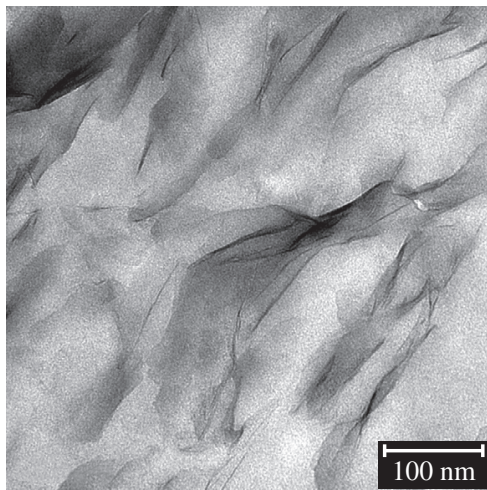

(d)

Figura 5. Fotomicrografias de MET para as amostras de nanocompósitos PVC/O-MMT obtidos pela incorporação da argila previamente esfoliada em DIDP/OSE a quente: a) e b) F5 (composto em pó); c) e d) F6 (composto granulado).

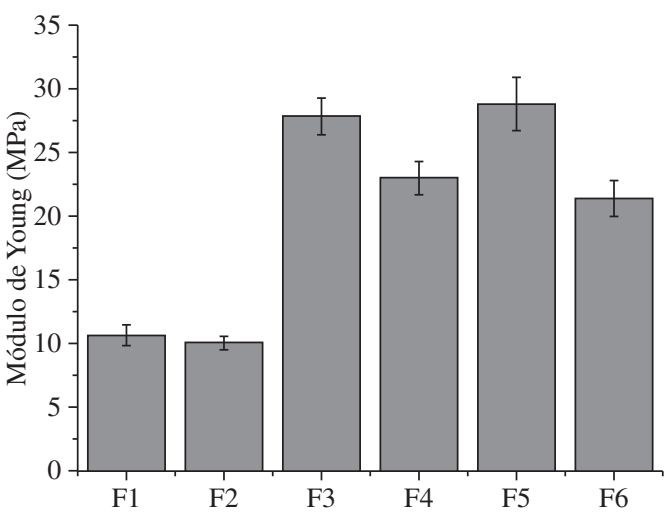

Figura 6. Módulo de Young das formulações de PVC/O-MMT avaliadas.

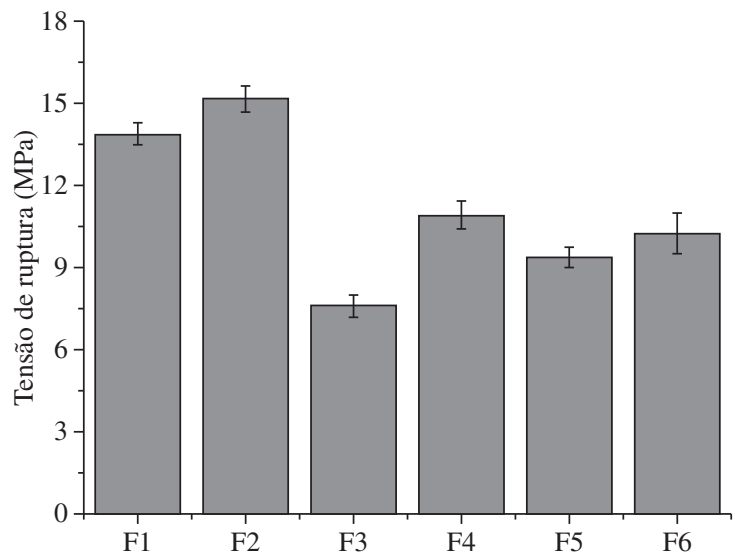

Figura 7. Tensão de ruptura das formulações de PVC/O-MMT avaliadas.

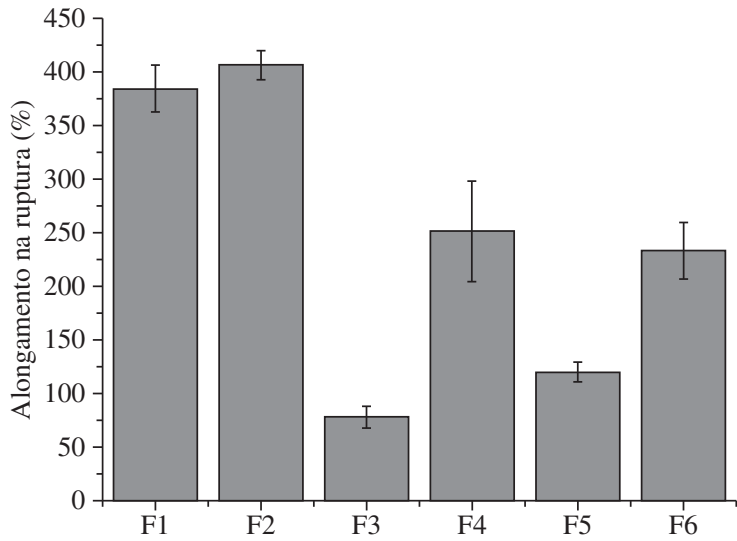

Figura 8. Alongamento na ruptura das formulações de PVC/O-MMT avaliadas.

Young, por conta do efeito de reforçamento, e redução da tensão de ruptura e alongamento na ruptura, indicativos de fragilização por conta da presença de inclusões em meio à matriz polimérica.

Uma análise detalhada dos resultados experimentais, de forma a identificar a significância estatística dos efeitos dos fatores processo de incorporação da O-MMT (direto no misturador e pré-esfoliado a quente) e morfologia final do composto (em pó ou granulado), foi realizada com o auxílio do software Minitab ${ }^{\circledR} 15$ (Minitab Inc., EUA). A análise de vari- 


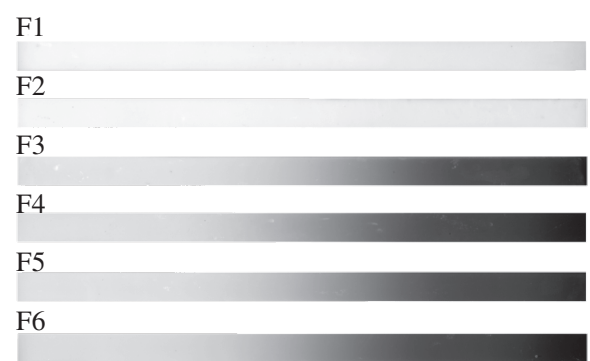

(a)

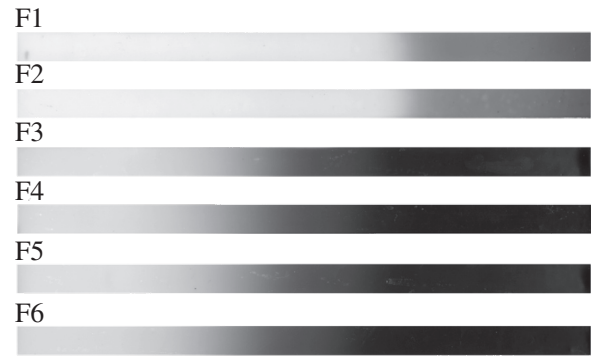

(b)

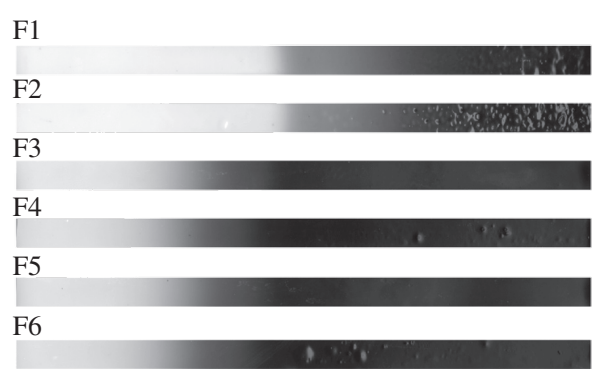

(c)

Figura 9. Resultados visuais de estabilidade térmica estática das formulações de PVC/O-MMT: a) 1 hora a $190{ }^{\circ} \mathrm{C}$; b) 1 hora a $210{ }^{\circ} \mathrm{C}$; e c) 1 hora a $230{ }^{\circ} \mathrm{C}$.

ância (ANOVA) dos resultados, em função dos fatores, mostrou que os efeitos do processo de incorporação da O-MMT não são estatisticamente significativos para nenhuma das propriedades de tração avaliadas, com valores da estatística $p$ variando entre 0,230 e 0,515 . No entanto, os efeitos da granulação do composto, como seria de se esperar, são bastante significativos para todas as propriedades medidas $(\mathrm{p}<0,001$, ou seja, mais de $99,9 \%$ de significância estatística para a diferença entre as médias), particularmente para a tensão de ruptura e alongamento na ruptura, indicativos de uma menor incidência de inclusões fragilizadoras na microestrutura dos nanocompósitos, que foram melhor processados e/ou homogeneizados.

A granulação dos compostos promove uma redução estatisticamente significativa no valor do módulo de Young das formulações granuladas, em comparação àquelas processadas diretamente do composto de PVC na forma de pó. Isto pode ser devido a uma maior interação entre a argila, melhor esfoliada, e a matriz polimérica, através da interface do sal de amônio quaternário. Uma vez que as formulações granuladas possuem maior área de interface com a O-MMT exposta, é possível que a lubricidade conferida pela cadeia alifática do sal de amônio quaternário reduza discretamente o valor do módulo de Young, em função da menor imobilização das cadeias do polímero no contato com a argila.

\section{Estabilidade térmica}

A Figura 9 apresenta os resultados do ensaio de estabilidade térmica estática em forno Metrastat a 190,210 e $230^{\circ} \mathrm{C}$. É possível verificar o mesmo padrão de comportamento nas três temperaturas avaliadas; ou seja, a incorporação da O-MMT afeta negativamente e de maneira significativa, a estabilidade térmica dos compostos de PVC, corroborando os efeitos de- letérios do sal de amônio quaternário no processo de desidrocloração, já observados por outros pesquisadores ${ }^{[2,22]}$.

A análise colorimétrica das fitas provenientes do forno Metrastat permite a determinação do onset de degradação, indicado através da mudança do patamar de $\Delta \mathrm{E} L * a * b *$ em relação à cor original de cada formulação, antes da exposição à temperatura de ensaio. Os dados de tempo de degradação foram tratados quanto à análise de variância, utilizando-se o software Minitab ${ }^{\circledR}$ 15. Assim foi confirmado que os efeitos dos fatores processo de incorporação da O-MMT e morfologia final do composto não são significativos do ponto de vista estatístico, uma vez que apresentam, respectivamente, valores de $\mathrm{p}=0,840$ e 0,704 , respectivamente.

\section{Conclusões}

Nanocompósitos de PVC/O-MMT, com microestrutura híbrida intercalada/parcialmente esfoliada, foram obtidos independentemente da forma de apresentação do composto (processado a partir do pó ou granulado) e do método de incorporação da O-MMT à resina de PVC (diretamente no misturador intensivo ou pré-esfoliada em DIDP/OSE a quente).

Medidas de DRX e observações de MET confirmam a microestrutura híbrida; a formação dos nanocompósitos foi também evidenciada pela alteração significativa das propriedades de tração das formulações. Neste caso, foi possível verificar que a granulação dos compostos beneficia a qualidade da microestrura do nanocompósito, uma vez que foi observada menor sensibilidade à fratura do mesmo.

Com relação à estabilidade térmica, confirmaram-se as conclusões de outros pesquisadores, que observaram piora da resistência à desidrocloração com a incorporação da O-MMT, devido à presença do sal de amônio quaternário. 
Este estudo permitiu ainda evidenciar que a granulação dos compostos não induz piora adicional nesta característica do composto, uma vez que não foram observadas diferenças estatisticamente significativas entre formulações processadas a partir do pó em comparação com os compostos granulados.

\section{Agradecimentos}

Os autores agradecem à Braskem S/A, através do programa NEO PVC (Núcleo de Estudos Orientados do PVC), pelo suporte dado a este projeto de pesquisa. $\mathrm{O}$ apoio técnico de Rafael Vilela Laurini, Lucas Agostinelli Polito e Mauro Oviedo, da Braskem S/A, e de Aline Taís da Rosa (MET) e Charles Dal Castel (DRX), da UFRGS, foram imprescindíveis à consecução deste trabalho.

\section{Referências Bibliográficas}

1. Chemical Market Associates - "2008 world vinyls analysis", Chemical Market Associates, Inc., Houston (2008).

2. Chow, G.-M. \& Kurihara, L. K. - "Chemical synthesis and processing of nanostructured powders and films", in: Nanostructured materials: Processing, properties and potential applications, C. C. Kock (ed.), Noyes Publications, Norwich (2002).

3. Jordan, J.; Jacob, K. I.; Tannenbaum, R.; Sharaf, M. A. \& Jasiuk, I. - Materials Science and Engineering: A, 393, p.1 (2004).

4. Schmidt, D.; Shah, D. \& Giannelis, E. P. - Current Opinion in Solid State \& Materials Science, 6, p.205 (2002).

5. Yang, F., Ou, Y. \& Yu, Z. - Journal of Applied Polymer Science, 69, p.355 (1998).

6. Gilman, J. W. - Applied Clay Science, 15, p.31 (1999).

7. Giannelis, E. P. - Materials and Design, 13, p.100 (1992).

8. Lan, T.; Kaviratna, P. D. \& Pinnavaia, T. J. - Chemistry of Materials, 6, p.573 (1994).

9. Wang, Z.; Lan, T. \& Pinnavaia, T. J. - Chemistry of Materials, 8, p.2200 (1996).

10. Alexandre, M. \& Dubois, P. - Materials Science and Engineering: R, 28, p.1 (2000).

11. Ray, S. S. \& Okamoto, M. - Progress in Polymer Science, 28, p.1539 (2003).

12. Paul, D. R. \& Robeson, L. M. - Polymer, 49, p.3187 (2008).
13. Pavlidou, S. \& Papaspyrides, C. D. - Progress in Polymer Science, 33, p.1119 (2008).

14. Utracki, L. A. - "Clay-containing polymeric nanocomposites”, v.1, Rapra Technology Ltd., Shropshire (2004).

15. Rodolfo, Jr., A. \& Mei, L. H. I. - Polímeros: Ciência e Tecnologia, 17, p.263 (2007).

16. Wang, D.; Parlow, D.; Yao, Q. \& Wilkie, C. A. - Journal of Vinyl and Additive Technology, 7, p.203 (2001).

17. Schaefer, D. W. \& Justice, R. S. - Macromolecules, 40, p.8501 (2007).

18. Yalcin, B. \& Cakmak, M. - Polymer, 45, p.6623 (2004).

19. Kovarova, L.; Kalendova, A.; Gerard, J.-F.; Malac, J.; Simonik, J. \& Weiss, Z. - Macromolecular Symposia, 221, p.105 (2005).

20. Peprnicek, T.; Duchet, J.; Kovarova, L.; Malac, J.; Gerard, J.-F. \& Simonik, J. - Polymer Degradation and Stability, 91, p.1855 (2006).

21. Peprnicek, T.; Kalendova, A.; Pavlova, E.; Simonik, J.; Duchet, J. \& Gerard, J.-F. - Polymer Degradation and Stability, 91, p.3322 (2006).

22. Francis, N. \& Schmidit, D. F. - "PVC/layered silicate nanocomposites: preparation, characterization, and properties”, in: ANTEC 2007, Cincinnati - EUA, p.1238 (2007).

23. Kalendova, A.; Kovarova, L.; Malac, Z.; Malac, J.; Vaculik, J.; Hrncirik, J. \& Simonik, J. - "Modified clay in polyvinyl chloride (PVC)”, in: ANTEC 2002, São Francisco - EUA (2002).

24. Southern Clay Products - "Cloisite ${ }^{\circledR}$ 30B - Typical physical properties bulletin”, disponível em www.scprod. com. Acesso em 4 fev. 2008.

25. Faulkner, P. G. - Journal of Macromolecular Science Physics B, 11, p.251 (1975).

26. Rabinovitch, E. B. \& Summers, J. - Journal of Vinyl Technology, 2, p.165 (1980).

27. Marques, R. P. \& Covas, J. A. - "Processing characteristics of U-PVC compounds", Companhia Industrial de Resinas Sintéticas, Estarreja (2003).

28. Fillot, L.-A.; Hajji, P.; Gauthier, C. \& Masenelli-Varlot, K. - Journal of Vinyl and Additive Technology, 12, p.98 (2006).

29. Alves, J. P. D. \& Rodolfo Jr., A. - Polímeros: Ciência e Tecnologia, 16, p.165 (2006).

30. Fornes, T. D.; Yoon, P. J.; Keskkula, H. \& Paul, D. R. Polymer, 42, p.9929 (2001). 